\title{
Initial Alignment of Point Clouds using Motors
}

\author{
Adam Leon Kleppe \\ Department of Mechanical and \\ Industrial Engineering, Norwegian \\ University of Science and Technology \\ $\mathrm{N}-7491$ \\ Trondheim, Norway \\ adam.l.kleppe@ntnu.no
}

\author{
Lars Tingelstad \\ Department of Mechanical and \\ Industrial Engineering, Norwegian \\ University of Science and Technology \\ N-7491 \\ Trondheim, Norway \\ lars.tingelstad@ntnu.no
}

\author{
Olav Egeland \\ Department of Mechanical and \\ Industrial Engineering, Norwegian \\ University of Science and Technology \\ $\mathrm{N}-7491$ \\ Trondheim, Norway \\ olav.egeland@ntnu.no
}

\begin{abstract}
This paper presents an approach for initial alignment or coarse registration of a partial 3D point cloud of objects. The method is based on computing the centroid of the points in the point cloud, and a line derived from the surface normals. This approach uses conformal geometric algebra and non-linear least squares optimization to achieve the results. The method performs well in experiments, and it is shown that it performs more accurately the more points are sampled.
\end{abstract}

\section{CCS CONCEPTS}

-Mathematics of computing $\rightarrow$ Nonlinear equations; $\cdot$ Computing methodologies $\rightarrow$ Optimization algorithms;

\section{KEYWORDS}

computer vision, conformal geometric algebra, initial alignment, screw theory

\section{ACM Reference format:}

Adam Leon Kleppe, Lars Tingelstad, and Olav Egeland. 2017. Initial Alignment of Point Clouds using Motors. In Proceedings of CGI '17, Yokohama, Japan, fune 27-30, 2017, 5 pages.

DOI: $10.1145 / 3095140.3097282$

\section{INTRODUCTION}

The 3D-3D registration problem [13] is a well-known problem in computer vision, and it is still a challenging problem. The problem is formulated as such: Assume that there are two sets of points, or point clouds

$$
\begin{array}{ll}
\boldsymbol{A}=\left\{\boldsymbol{a}_{i}\right\}, & \boldsymbol{a}_{i} \in \mathbb{R}^{3,1}, i=1, \ldots, m \\
\boldsymbol{B}=\left\{\boldsymbol{b}_{j}\right\}, & \boldsymbol{b}_{j} \in \mathbb{R}^{3,1}, j=1, \ldots, n
\end{array}
$$

Find the rotation $R \in S O(3)$ and the translation $t \in \mathbb{R}^{3}$ that gives the most optimal alignment between the two sets.

$$
\min \sum_{i}\left\|\boldsymbol{b}_{j^{*}}-\boldsymbol{R} \boldsymbol{a}_{i}-\boldsymbol{t}\right\|^{2}
$$

Permission to make digital or hard copies of all or part of this work for personal or classroom use is granted without fee provided that copies are not made or distributed for profit or commercial advantage and that copies bear this notice and the full citation on the first page. Copyrights for components of this work owned by others than ACM must be honored. Abstracting with credit is permitted. To copy otherwise, or republish, to post on servers or to redistribute to lists, requires prior specific permission and/or a fee. Request permissions from permissions@acm.org.

CGI '17, Yokohama, Japan

(C) 2017 ACM. 978-1-4503-5228-4/17/06 ..\$15.00

DOI: $10.1145 / 3095140.3097282$ where $\boldsymbol{b}_{j^{*}}$ is the optimal corresponding point to $\boldsymbol{a}_{i}$, based on distance given the optimal $R$ and $\boldsymbol{t}$.

This problem can be divided into two sub-problems. The first is to find the pose that aligns the two point clouds, and the second is to find point-wise correspondences between the sets, or in other words, which point in $\boldsymbol{A}$ corresponds to the points in $\boldsymbol{B}$, or even if there is a correspondence.

A large number of methods have been proposed to solve the registration problem $[14,19]$. The most popular approach is ICP [2, $5,15]$. These methods can be classified as either coarse or fine registration methods, and usually both have to be applied in order to get globally optimal solution to the registration problem. Here the coarse registration aims to find a rough initial alignment which improves the initial conditions for the fine registration.

Most of the fine registration methods, including ICP, are so called Expectation-Maximization algorithms[13], because they alternate between solving the two sub-problems until both reaches a local minima. A known restriction with EM-algorithms is that they only find local optimal solutions. This means that in order for them to converge to the global optimum, the algorithm either has to be expanded to include global optimization techniques, such as GoICP [23] or Sparse ICP [3], or it has to have good initial conditions, i.e. the point clouds have to have a good initial alignment relative to each other, in order to converge to the correct solution. This is achieved with coarse registration methods, such as [7, 16-18].

The coarse registration methods usually only solves one of the sub-problems: Finding the pose that aligns the two point clouds. This means that the methods does not take the point correspondences into account. The most common approach is to create a set of features or signatures in each point cloud, and search for correspondences between the features. Examples of this are Point Signatures [6], Spin Images [12], Point Feature Histograms [16, 17], and Principal Component Analysis [7].

Both Point Signatures, Spin Images and Point Feature Histograms use techniques that originated from $2 \mathrm{D}$ computer vision. They use some measurement using relative distances and angles to generate features. These measurements are calculated from the points and surface normals of the point cloud. The methods uses different schemes to categorize the features, be it sets, tables or histograms to group them into cells. The main drawback with these methods is that the accuracy of the them depend on the resolution of these cells.

In this paper we propose a new method for initial alignment of two point clouds, i.e. coarse registration. The method constructs a feature using the centroid and a line computed from the surface normals of a point cloud. This feature is calculated using Conformal 
Geometric Algebra. The motor which aligns the features of two point clouds is found using Non-Linear Least Square Optimization, and results in an initial alignment pose between the two point clouds. The benefit of optimization techniques to find the pose is that the accuracy does not depend on a given resolution. This method also has the benefit of requiring less computation than the methods mentioned above.

This paper is is organised as follows: Section 2 is the preliminaries, which introduces the parts of Conformal Geometric Algebra used in the paper. Section 3 describes the proposed method. Section 4 show the conducted experiment and the results, and lastly the conclusion is found in Section 5.

\section{PRELIMINARIES}

\subsection{Conformal Geometric Algebra}

The geometric algebra of the Euclidean space $\mathbb{R}^{3}$ is denoted $\mathbb{R}_{3}$, while the conformal model of geometric algebra is denoted $\mathbb{R}_{4,1}$ resulting in the null basis $\left\{e_{0}, e_{1}, e_{2}, e_{3}, e_{\infty}\right\}[9,10]$. The basis vector $e_{\infty}$ represents the point at infinity, while $e_{0}$ represents an arbitrary origin. These basis vectors have the properties $e_{\infty}^{2}=e_{0}^{2}=0$ and $e_{\infty} \cdot e_{0}=-1$. The notation $\mathbb{R}_{3}^{k}$ refers to the $k$-grade elements of $\mathbb{R}_{3}$. The highest grade element of $\mathbb{R}_{3}$, is the Euclidean pseudoscalar, which is denoted $I_{3}$. The conformal pseudoscalar is denoted $I$. The conformal dual of a multivector $X$ is denoted $X^{*}=X I^{-1}$.

Euclidean vectors $\boldsymbol{p} \in \mathbb{R}_{3}$ maps to points $\boldsymbol{P} \in \mathbb{R}_{4,1}$ using

$$
\boldsymbol{P}=\boldsymbol{p}+\frac{1}{2} \boldsymbol{p}^{2} e_{\infty}+e_{0}
$$

A line $\boldsymbol{\ell} \in \mathbb{R}_{4,1}^{3}$ is constructed as the outer product of two conformal points and the point at infinity:

$$
\boldsymbol{\ell}=\boldsymbol{P}_{A} \wedge \boldsymbol{P}_{B} \wedge e_{\infty}
$$

This can be expressed as

$$
\boldsymbol{\ell}=\left(\boldsymbol{p}+e_{0}\right) \wedge \hat{\boldsymbol{n}} \wedge e_{\infty}
$$

where $\boldsymbol{p}$ is the Euclidean point and $\hat{\boldsymbol{n}}$ is the Euclidean directional vector of the line. This is called the direct representation in [9], and the OPNS representation in [10].

The dual representation of a line in conformal space is

$$
\boldsymbol{\ell}^{*}=\boldsymbol{A}+\boldsymbol{b} e_{\infty}
$$

where $\boldsymbol{A}=\hat{\boldsymbol{n}}^{*}$ is the directional bivector, and $\boldsymbol{b}$ is the momentum of the line. It is noted that $\boldsymbol{A} \wedge \boldsymbol{b}=0$.

A screw $S$, is a line with a pitch, meaning that

$$
S^{*}=A+b e_{\infty}
$$

where $\boldsymbol{A} \wedge \boldsymbol{b}$ can be an arbitrary number.

A screw can be further described as

$$
S^{*}=\boldsymbol{A}+\left(\boldsymbol{b}_{\|}+\boldsymbol{b}_{\perp}\right) e_{\infty}
$$

where $\boldsymbol{A} \wedge \boldsymbol{b}_{\|}=0$, meaning that $\boldsymbol{A}+\boldsymbol{b}_{\|} e_{\infty}$ is a dual line and $\boldsymbol{b}_{\perp} e_{\infty}$ is the pitch.

A screw is generated by adding two lines together. This is shown when

$$
S^{*}=\boldsymbol{\ell}_{1}^{*}+\boldsymbol{\ell}_{2}^{*}=\boldsymbol{A}_{1}+\boldsymbol{A}_{2}+\left(\boldsymbol{b}_{1}+\boldsymbol{b}_{2}\right) e_{\infty}
$$

where $A_{1} \wedge b_{1}=A_{2} \wedge b_{2}=0$, but $A_{1} \wedge b_{2}$ and $A_{2} \wedge b_{1}$ cannot be guaranteed zero. This also holds for the addition of multiple lines.

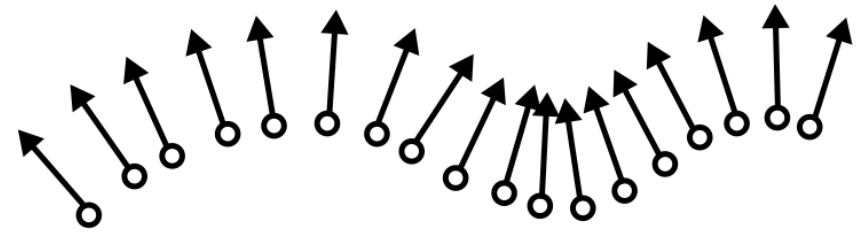

Figure 1: 2D Representation of the points in a point cloud and their respective surface normal

A flag [20] can be written as the sum of a line and a conformal point

$$
f=\ell+P
$$

\section{METHOD}

This method uses two point clouds, which represents the surface of a given object, and finds the motor $\boldsymbol{M}$ which is an optimal alignment between the two point clouds. This is achieved by finding the motor which minimizes the error between the centroids of each point cloud, which results in an optimal translation, and at the same time minimizes the deviation of the average line.

Assume that two point clouds $X$ and $Y$ are given by a set of points and their respective surface normals.

$$
\begin{array}{cl}
X=\left\{\boldsymbol{x}_{i}, \hat{\boldsymbol{n}}_{x_{i}}\right\}, & \boldsymbol{x}_{i}, \hat{\boldsymbol{n}}_{x_{i}} \in \mathbb{R}^{3,1},\left\|\hat{\boldsymbol{n}}_{x_{i}}\right\|_{2}=1, i=1, \ldots, m \\
Y=\left\{\boldsymbol{y}_{j}, \hat{\boldsymbol{n}}_{y_{j}}\right\}, & \boldsymbol{y}_{j}, \hat{\boldsymbol{n}}_{y_{j}} \in \mathbb{R}^{3,1},\left\|\hat{\boldsymbol{n}}_{y_{j}}\right\|_{2}=1, j=1, \ldots, n
\end{array}
$$

Note that the number of points in $X$ and $Y$ are not the same, and that $\boldsymbol{x}_{i}$ and $\boldsymbol{y}_{j}$ do not necessarily correspond if $i=j$. It is assumed that the surface normals are either calculated from the CAD model, or by estimating it using the points in the point cloud data.

\subsection{Centroid}

The conformal centroid of each point cloud is found by

$$
\begin{aligned}
& \overline{\boldsymbol{P}}_{X}=C\left(\frac{1}{m} \sum_{i=1}^{m} \boldsymbol{x}_{i}\right) \\
& \overline{\boldsymbol{P}}_{Y}=C\left(\frac{1}{n} \sum_{j=1}^{n} \boldsymbol{y}_{j}\right)
\end{aligned}
$$

where $C(\boldsymbol{p})$ is the conformal point based on the Euclidean vector $\boldsymbol{p}$.

\subsection{Average of lines}

The average of a set of lines is constructed as a screw which is the sum of all lines, where each line is generated from a point and its surface normal.

$L_{X}$ and $L_{Y}$ are the sets containing all lines generated from the point cloud.

$$
\begin{aligned}
& \boldsymbol{L}_{X}=\left\{\boldsymbol{\ell}_{x_{i}}=\left(\boldsymbol{x}_{i}+n_{0}\right) \wedge \hat{\boldsymbol{n}}_{x_{i}} \wedge n_{\infty}\right\} \\
& \boldsymbol{L}_{Y}=\left\{\boldsymbol{\ell}_{y_{j}}=\left(\boldsymbol{y}_{j}+n_{0}\right) \wedge \hat{\boldsymbol{n}}_{y_{i}} \wedge n_{\infty}\right\}
\end{aligned}
$$

The sum of these lines become a screw, where the screw axis is computed as the average of the lines. The point cloud, generated lines, average line and centroid can be viewed in Figure 2. It can be 


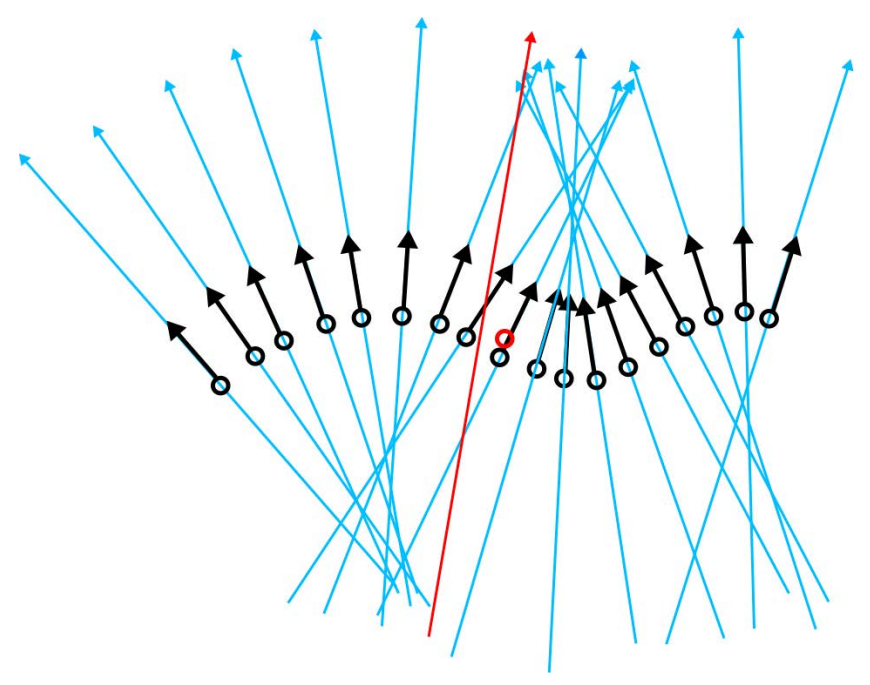

Figure 2: Lines generated from each point in the point cloud. The red line represents the average line, while the red point is the centroid

seen here that the screw axis does not necessarily pass through the centroid.

The screws of the two point clouds, $X$ and $Y$, are defined as

$$
\begin{aligned}
& S_{X}=\frac{1}{n} \sum_{i=1}^{n} \boldsymbol{\ell}_{x_{i}}^{*} \\
& S_{Y}=\frac{1}{m} \sum_{j=1}^{m} \boldsymbol{\ell}_{y_{j}}^{*}
\end{aligned}
$$

It can be seen that these screws are the average of all lines since

$$
\begin{aligned}
S & =\frac{1}{n} \sum_{i=1}^{n} \boldsymbol{\ell}_{i}^{*} \\
& =\frac{1}{n} \sum_{i=1}^{n} \boldsymbol{A}_{\boldsymbol{i}}+\boldsymbol{b}_{i} e_{\infty} \\
& =\frac{1}{n}\left(\sum_{i=1}^{n} \boldsymbol{A}_{\boldsymbol{i}}+\sum_{i=1}^{n} \boldsymbol{b}_{i} e_{\infty}\right) \\
& =\bar{A}+\overline{\boldsymbol{b}} e_{\infty}
\end{aligned}
$$

where $\bar{A}$ is the average directional bivector, and $\bar{b}$ is the average momentum. This can be written as

$$
S=\bar{A}+\left(\overline{\boldsymbol{b}}_{\|}+\overline{\boldsymbol{b}}_{\perp}\right) e_{\infty}
$$

where $\bar{A} \wedge \overline{\boldsymbol{b}}_{\|}=0$.

This can be rewritten using the average line $\overline{\boldsymbol{\ell}}$, which is the average of the combined lines.

$$
\begin{aligned}
S & =\overline{\boldsymbol{A}}+\left(\overline{\boldsymbol{b}}_{\|}+\overline{\boldsymbol{b}}_{\perp}\right) e_{\infty} \\
& =\overline{\boldsymbol{A}}+\overline{\boldsymbol{b}}_{\|} e_{\infty}+\overline{\boldsymbol{b}}_{\perp} e_{\infty} \\
& =\overline{\boldsymbol{l}}^{*}+\overline{\boldsymbol{b}}_{\perp} e_{\infty}
\end{aligned}
$$

\subsection{Restrictions}

There are in total 7 degrees of freedom in a motion, 3 to translation, 3 to rotation and 1 to scale [11]. This method uses only one line and one point is known in both point clouds and therefore in both coordinate systems. This forces seven constraints upon the system, 4 from the line and 3 from the point. This means the method is able to perform any rigid-body motion.

Since each line is generated by a point $\boldsymbol{p}$ and its corresponding surface normal $\hat{\boldsymbol{n}}$, these lines can be described as a force $\boldsymbol{F}=\hat{\boldsymbol{n}}$ at $\boldsymbol{p}$, where $|\boldsymbol{F}|=1$. To sum all these forces is the same as evaluating the force applied over the whole surface area, which by definition is the same as pressure.

This forces a restriction upon the method: This method cannot be used on the whole surface of an object. This is because the pressure over an enclosed surface area is zero. This means that if the whole surface is sampled by points and these points generate lines, then the sum of these lines will be zero.

As mentioned, this method is used on point clouds generated from the viewable surfaces of an object. In practice, this means that this restriction will never occur, since the whole surface of an object cannot be viewed at the same time.

\subsection{Motor Estimation}

Each point cloud, $X$ and $Y$, have one centroid, $\bar{P}_{X}$ and $\bar{P}_{Y}$, and one screw axis, $\overline{\boldsymbol{\ell}}_{X}$ and $\overline{\boldsymbol{\ell}}_{Y}$.

From these, their respective flags are defined as

$$
\begin{array}{r}
f_{X}=\overline{\boldsymbol{\ell}}_{X}+\overline{\boldsymbol{P}}_{X} \\
f_{Y}=\overline{\boldsymbol{\ell}}_{Y}+\overline{\boldsymbol{P}}_{Y}
\end{array}
$$

In order to minimize the error between these two flags, we can find the optimal motor between them.

$$
\begin{aligned}
& f_{X}-\boldsymbol{M}^{\dagger} \boldsymbol{f}_{Y} \tilde{\boldsymbol{M}}^{\dagger} \\
& \overline{\boldsymbol{\ell}}_{X}-\boldsymbol{M}^{\dagger} \overline{\boldsymbol{\ell}}_{Y} \tilde{\boldsymbol{M}}^{\dagger}+\overline{\boldsymbol{P}}_{X}-\boldsymbol{M}^{\dagger} \overline{\boldsymbol{P}}_{Y} \tilde{\boldsymbol{M}}^{\dagger}=0
\end{aligned}
$$

where $\boldsymbol{M}^{\dagger}$ is the optimal motor between the two point clouds. The result of zero is only possible if both point clouds are identical.

When comparing two point clouds which are not identical, either because of added noise or different points are used, an optimization scheme could be used. The motor which is the optimal transform between the two flags, will have one unique solution, since two lines and two points are used in the minimization function $[4,8]$.

\subsection{Error Functions}

The optimization of the motor between the two centroids in the flag only require an error measurement in the form of distance. The distance measure between the two centroids is easily found with

$$
\epsilon_{\boldsymbol{P}}=d^{2}=\overline{\boldsymbol{P}}_{X} \cdot \boldsymbol{M} \overline{\boldsymbol{P}}_{Y} \tilde{\boldsymbol{M}}
$$

where $\epsilon_{P}$ is the error function for the two centroids.

For the average lines in the flag, both the distance and the angle has to be optimized [1]. These parameters can be extracted from the motor Mwhich transforms one to the other.

$$
\boldsymbol{M}=\frac{\overline{\boldsymbol{\ell}}_{X}}{\overline{\boldsymbol{\ell}}_{Y}}
$$


where

$\boldsymbol{M}=\cos \theta-\sin \theta \boldsymbol{A}_{n}-\sin \theta \boldsymbol{b}_{n} e_{\infty}-d \cos \theta \boldsymbol{a}_{n} e_{\infty}+d \sin \theta e_{1} e_{2} e_{3} e_{\infty}$

where $A_{n}=\boldsymbol{a}_{n}^{*}$ and $\boldsymbol{b}_{n}$ are components of the common normal line $\boldsymbol{\ell}_{n}$.

According to [22], a good error function for angles is $\sin \frac{\theta}{2}$. This is because the error function is at most $\sin \frac{\pi}{2}=1$, which means that outliers do not have a large error, but are bound by 1 . This can be calculated using

$$
\frac{1}{2}(1-\cos \theta)=\sin ^{2} \frac{\theta}{2}
$$

The distance between the two lines can be calculated by decomposing the motor in a different manner:

$$
M=T R
$$

where

$$
\begin{aligned}
& T=1-\frac{1}{2} \boldsymbol{t} e_{\infty} \\
& R=-e_{0} \cdot\left(M e_{\infty}\right)
\end{aligned}
$$

where $T$ is the translation and $R$ is the rotation.

The distance between the lines can then be calculated using

$$
\begin{array}{r}
\delta=\|\delta\| \\
\delta=\frac{t \wedge B}{B}
\end{array}
$$

where

$$
\begin{gathered}
\boldsymbol{t}=-2 \frac{e_{0} \cdot \boldsymbol{M}}{\boldsymbol{R}} \\
\boldsymbol{B}=\frac{\langle\boldsymbol{R}\rangle_{2}}{\left\|\langle\boldsymbol{R}\rangle_{2}\right\|}
\end{gathered}
$$

where $\langle\boldsymbol{R}\rangle_{2}$ is the 2-blade component of $\boldsymbol{R}$.

With these parameters, the error function $\epsilon_{\bar{\ell}}$ can be formulated as

$$
\epsilon_{\overline{\boldsymbol{\ell}}}=\delta^{2}+\sin ^{2} \frac{\theta}{2}=\delta^{2}+\frac{1}{2}(1-\cos \theta)
$$

\subsection{Non-linear Least Square Optimization}

The non-linear least square optimization solver was used to estimate the motor. By using the given error functions, the equation to be minimized is given by

$$
\min \frac{1}{2}\left(\epsilon_{\overline{\boldsymbol{\ell}}}+\epsilon_{\boldsymbol{p}}\right)^{2}=\min \frac{1}{2}\left(\delta^{2}+\sin ^{2} \frac{\theta}{2}+d^{2}\right)^{2}
$$

such that

$$
\boldsymbol{M} \tilde{M}=1
$$

which is solved using Levenberg-Marquardt algorithm which is has been developed to work for conformal points and lines. The algorithm is presented in [21].
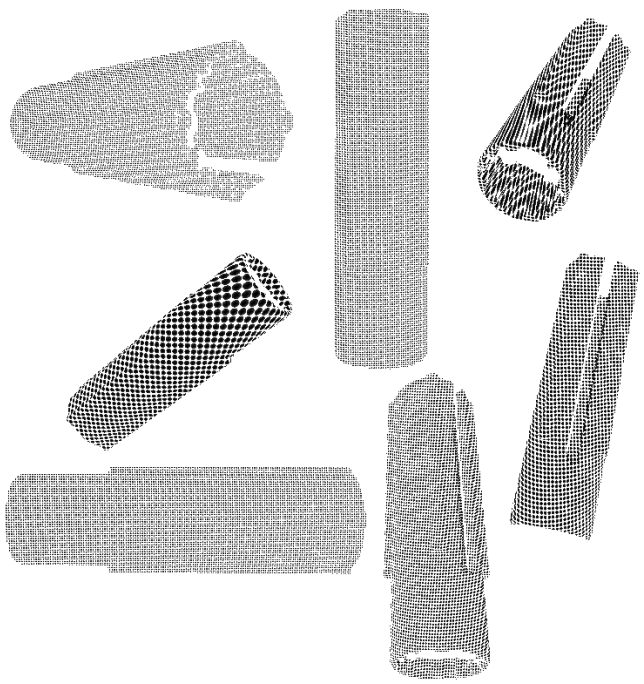

Figure 3: Seven viewpoint samples generated from one of the objects.

\section{EXPERIMENTS}

Several point clouds were generated from a set of CAD models. Since the method only works when having partial surfaces, the point clouds were generated using viewpoint sampling.

Viewpoint sampling was done by placing a virtual camera facing the 3D model. The points are generated based on the surfaces that are visible by the virtual camera. In order to get the whole view of the CAD model, several samples were generated from different viewpoints. The position of the viewpoints were calculated using a tessellated sphere which surrounded the 3D model. Each vertex of the tessellated sphere was set as a viewpoint.

Two models each generated 3 point clouds per viewpoint, one with 100 points, one with 1000 points and one with 10000 points. There were a total of 42 viewpoints per object, resulting in a total of 252 point clouds. A sample of these point clouds is shown in Figure 3. Each point cloud was given an arbitrary transformation and noise was applied to the point cloud. The initial alignment method together with the GAME framework was used to calculate the transformation between the two point clouds. The error was calculated by applying the Root Mean Square between the points in each point cloud, and is measured in meters.

$$
\epsilon=\sqrt{\frac{1}{n} \boldsymbol{P}_{X_{1}} \cdot \boldsymbol{M} \boldsymbol{P}_{Y_{2}} \tilde{\boldsymbol{M}}+\boldsymbol{P}_{X_{2}} \cdot \boldsymbol{M} \boldsymbol{P}_{Y_{2}} \tilde{\boldsymbol{M}} \ldots \boldsymbol{P}_{X_{n}} \cdot \boldsymbol{M} \boldsymbol{P}_{Y_{n}} \tilde{\boldsymbol{M}}}
$$

The result of these tests are shown in Table 1. It can be seen from the table that the error decreased as the number of samples increased. This is expected since the more samples are used, the more the average will cancel out the added noise. The average error was $6.8325 \times 10^{-4} \mathrm{~m}$ for a 100 samples, $3.323 \times 10^{-4} \mathrm{~m}$ for a 1000 samples and $3.7214 \times 10^{-5} \mathrm{~m}$ for a 10000 samples.

\section{CONCLUSION}

This paper shows a method for initial alignment for point clouds. The method finds the optimal motor between the centroid and 


\begin{tabular}{|c|c|c|c|c|c|c|}
\hline & \multicolumn{2}{|c|}{ 100 Samples } & \multicolumn{2}{c|}{ 1000 Samples } & \multicolumn{2}{c|}{10000 Samples } \\
\hline Point Cloud & \# Iteration & Error [m] & \# Iteration & Error [m] & \# Iteration & Error [m] \\
\hline 1 & 6 & $1.840 \times 10^{-3}$ & 6 & $9.4128 \times 10^{-3}$ & 17 & $6.6647 \times 10^{-7}$ \\
6 & 7 & $4.8510 \times 10^{-4}$ & 16 & $1.3453 \times 10^{-6}$ & 12 & $3.5899 \times 10^{-7}$ \\
7 & 7 & $3.1998 \times 10^{-4}$ & 14 & $2.5855 \times 10^{-6}$ & 12 & $2.5160 \times 10^{-6}$ \\
14 & 7 & $1.8475 \times 10^{-4}$ & 7 & $5.7251 \times 10^{-3}$ & 12 & $1.0650 \times 10^{-8}$ \\
22 & 7 & $1.1063 \times 10^{-3}$ & 6 & $1.2058 \times 10^{-2}$ & 11 & $3.9998 \times 10^{-8}$ \\
31 & 6 & $1.7973 \times 10^{-3}$ & 7 & $5.5537 \times 10^{-3}$ & 7 & $1.0105 \times 10^{-2}$ \\
45 & 7 & $1.0676 \times 10^{-3}$ & 13 & $4.3508 \times 10^{-9}$ & 11 & $8.0189 \times 10^{-8}$ \\
65 & 7 & $1.1544 \times 10^{-4}$ & 13 & $2.5538 \times 10^{-9}$ & 13 & $8.5678 \times 10^{-9}$ \\
73 & 12 & $8.7525 \times 10^{-9}$ & 11 & $7.6853 \times 10^{-9}$ & 10 & $4.7666 \times 10^{-9}$ \\
78 & 11 & $1.0848 \times 10^{-8}$ & 11 & $3.4762 \times 10^{-8}$ & 12 & $6.9853 \times 10^{-9}$ \\
\hline Average & & $6.8325 \times 10^{-4}$ & & $3.323 \times 10^{-4}$ & & $3.7214 \times 10^{-5}$ \\
\hline
\end{tabular}

Table 1: Sample of the result of the initial alignment method. The number of iterations before the method terminated and the resulting error from the true transform is shown. The average of all results is also shown.

average of lines of two point clouds, which is the initial alignment. The average error was $6.8325 \times 10^{-4} \mathrm{~m}$ for a 100 samples, $3.323 \times 10^{-4} \mathrm{~m}$ for a 1000 samples and $3.7214 \times 10^{-5} \mathrm{~m}$ for a 10000 samples.

\section{REFERENCES}

[1] Eduardo Bayro-Corrochano, Kostas Daniilidis, and Gerald Sommer. 2000. Motor algebra for 3D kinematics: the case of the hand-eye calibration. Fournal of Mathematical Imaging and Vision 13, 2 (2000), 79-100. DOI : https://doi.org/10. 1023/A:1026567812984

[2] Paul Besl and Neil McKay. 1992. A Method for Registration of 3-D Shapes. (1992), 239-256 pages. DOI : https://doi.org/10.1109/34.121791

[3] Sofien Bouaziz, Andrea Tagliasacchi, and Mark Pauly. 2013. Sparse iterative closest point. Computer Graphics Forum 32, 5 (2013), 113-123. DOI : https://doi. org $/ 10.1111 /$ cgf. 12178

[4] H.H. Chen. 1991. A screw motion approach to uniqueness analysis of head-eye geometry. Proceedings. 1991 IEEE Computer Society Conference on Computer Vision and Pattern Recognition (1991), 145-151. DOI : https://doi.org/10.1109/CVPR.1991. 139677 arXiv:cs/9605103

[5] Y. Chen and G. Medioni. 1991. Object modeling by registration of multiple range images. Proceedings. 1991 IEEE International Conference on Robotics and Automation April (1991), 2724-2729. DOI : https://doi.org/10.1109/ROBOT.1991. 132043

[6] Chin Seng Chua and Ray Jarvis. 1997. Point Signatures: A New Representation for 3D Object Recognition. International fournal of Computer Vision 25, 1 (1997), 63-85. DOI : https://doi.org/10.1023/A:1007981719186

[7] Do Hyun Chung, Il Dong Yun, and Sang Uk Lee. 1997. Registration of Multiple Range Views using the Reverse Calibration Technique. (1997), 0-19.

[8] Kostas Daniilidis. 1999. Hand-Eye Calibration Using Dual Quaternions. The International fournal of Robotics Research 18, 3 (1999), 286-298. DOI : https://doi org/10.1177/02783649922066213

[9] Leo Dorst, Daniel Fontijne, and Stephen Mann. 2009. Geometric Algebra for Computer Science: An Object-Oriented Approach to Geometry. Morgan Kaufmann Publishers Inc. San Francisco, CA, USA. 664 pages. http://www.geometricalgebra. net/

[10] Dietmar Hildenbrand. 2013. Foundations of Geometric Algebra Computing. Geometry and Computing, Vol. 8. Springer Berlin Heidelberg, Berlin, Heidelberg. DOI : https://doi.org/10.1007/978-3-642-31794-1

[11] Berthold K P Horn. 1987. Closed-form solution of absolute orientation using unit quaternions. Journal of the Optical Society of America A 4, 4 (1987), 629. DOI : https://doi.org/10.1364/JOSAA.4.000629

[12] Andrew E. Johnson and Martial Hebert. 1999. Using spin images for efficient object recognition in cluttered 3D scenes. IEEE Transactions on Pattern Analysis and Machine Intelligence 21, 5 (1999), 433-449. DOI : https://doi.org/10.1109/34. 765655

[13] Hongdong Li and Richard Hartley. 2007. The 3D-3D registration problem revisited Proceedings of the IEEE International Conference on Computer Vision (2007). DOI : https://doi.org/10.1109/ICCV.2007.4409077

[14] Helmut Pottmann, Stefan Leopoldseder, and Michael Hofer. 2004. Registration without ICP. Computer Vision and Image Understanding 95, 1 (2004), 54-71. DOI : https://doi.org/10.1016/j.cviu.2004.04.002
[15] S. Rusinkiewicz and M. Levoy. 2001. Efficient variants of the ICP algorithm. Proceedings of International Conference on 3-D Digital Imaging and Modeling, 3DIM 2001-Janua (2001), 145-152. DOI : https://doi.org/10.1109/IM.2001.924423

[16] Radu Bogdan Rusu, Nico Blodow, and Michael Beetz. 2009. Fast Point Feature Histograms (FPFH) for 3D registration. IEEE International Conference on Robotics and Automation (2009), 3212-3217. DOI: https://doi.org/10.1109/ROBOT.2009. 5152473

[17] Radu Bogdan Rusu, Gary Bradski, Romain Thibaux, and John Hsu. 2010. Fast 3D Recognition and Pose Using the Viewpoint Feature Histogram. (2010), 21552162.

[18] B Sabata and J. K. Aggarwal. 1996. Surface Correspondence and Motion Computation from a Pair of Range Images. Computer Vision and Image Understanding 63, 2 (1996), 232-250. DOI : https://doi.org/10.1006/cviu.1996.0017

[19] Joaquim Salvi, Carles Matabosch, David Fofi, and Josep Forest. 2007. A review of recent range image registration methods with accuracy evaluation. Image and Vision Computing 25, 5 (2007), 578-596. DOI : https://doi.org/10.1016/j.imavis. 2006.05.012

[20] J. M. Selig. 2005. Geometric Fundamentals of Robotics. 398 pages. DOI: https: //doi.org/10.1007/b138859 arXiv:arXiv:1011.1669v3

[21] Lars Tingelstad and Olav Egeland. 2016. Motor Estimation using Heterogeneous Sets of Objects in Conformal Geometric Algebra. Advances in Applied Clifford Algebras (2016), 1-15. DOI : https://doi.org/10.1007/s00006-016-0692-8

[22] Robert Valkenburg and Leo Dorst. 2011. Estimating Motors from a Variety of Geometric Data in 3D Conformal Geometric Algebra. Guide to Geometric Algebra in Practice XVII, December (2011), 25-45. DOI : https://doi.org/10.1007/ 978-0-85729-811-9 arXiv:arXiv:1011.1669v3

[23] Jiaolong Yang, Hongdong Li, and Yunde Jia. 2013. Go-ICP: Solving 3D registration efficiently and globally optimally. Proceedings of the IEEE International Conference on Computer Vision (2013), 1457-1464. DOI : https://doi.org/10.1109/ICCV.2013. 184 